\title{
Inguinal abscess in twin infants
}

\author{
Meghmala Sadhukhan ${ }^{1}$, Baikuntha N. Mishra ${ }^{2 *}$, Ranjit K. Joshi ${ }^{1}$, Prabin Prakash Pahi ${ }^{1}$
}

\author{
${ }^{1}$ Department of Paediatrics and Neonatology, AMRI Hospitals, Bhubaneswar, Odisha, India \\ ${ }^{2}$ Department of Paediatric Surgery, AMRI Hospitals, Bhubaneswar, Odisha, India
}

Received: 22 February 2020

Accepted: 07 April 2020

\section{*Correspondence:}

Dr. Baikuntha N. Mishra,

E-mail: mbaikuntha@gmail.com

Copyright: (c) the author(s), publisher and licensee Medip Academy. This is an open-access article distributed under the terms of the Creative Commons Attribution Non-Commercial License, which permits unrestricted non-commercial use, distribution, and reproduction in any medium, provided the original work is properly cited.

\begin{abstract}
Inguinal abscess is uncommon in infants and children. Majority of cases in the infantile period is primary and they present as leg or groin swelling, limitation of limb movement and pain. The source of microorganism is from the haematogenous spread of normal flora of the skin due to immature immune system. Ultrasound is the investigation of choice which confirms the diagnosis and delineates the areas of collection. Antibiotic alone is insufficient in most cases and drainage is required. Delayed treatment and inadequate drainage carry a high risk of mortality and sequelae due to damage to the underlying joint. We present 2 cases of primary inguinal abscess in twin infants who presented at 1 month and 2 months of age correspondingly, in whom early diagnosis and management lead to quick recovery.
\end{abstract}

Keywords: Infant, Inguinal abscess, Twin

\section{INTRODUCTION}

Inguinal abscess is uncommon in infants and children. ${ }^{1-3}$ It can be primary which is idiopathic or has no detectable source and secondary in which the source of infection is direct spread from adjacent structures. ${ }^{1-3}$ Majority of inguinal abscess in the infantile period is primary. ${ }^{1,2}$ The proposed hypothesis in case of primary abscess is that the normal flora of the skin spreads haematogenously due to immature immune system in infants. ${ }^{1-3}$ The most common organism seen in primary inguinal abscess is Staphylococcus aureus. ${ }^{1,2}$ In case of secondary inguinal abscess common organisms implicated are Staphylococcus aureus, Streptococcus pneumoniae and Escherichia coli..$^{1,2}$

We present 2 cases of twin infants who presented with similar complaints consecutively with the diagnosis of inguinal abscess in whom early diagnosis let to quick and complete recovery. In one of the infants the organism isolated was Staphylococcus hominis which has not been reported previously to be isolated in cases of inguinal abscess.

\section{CASE REPORT}

\section{Case 1}

A 1-month old male baby presented with left groin swelling for 4 days. Current weight was $2.1 \mathrm{Kg}$. This baby was the $2^{\text {nd }}$ twin of twin siblings born at 34 weeks gestation. He was admitted to the neonatal unit initially for LBW and respiratory distress after birth for 48 hours which settled with oxygen and orogastric tube feeding. On examination, there was an inguinal swelling of $4 \times 4$ $\mathrm{cm}$ size with redness and tenderness. Ultrasound showed small cystic lesion $18 \times 11 \mathrm{~mm}$ in left parietal wall over inguinal region suggestive of necrotic node/abscess. Blood investigations showed raised total WBC count of $21,600 / \mathrm{mm}^{3}$. IV antibiotics were started with IV Meropenem and Teicoplanin. Drainage of abscess was done under GA and pus was sent for culture study which grew Staphylococcus hominis. Blood culture was negative. Baby improved clinically. IV antibiotics were given for 7 days and then changed to oral as per sensitivity pattern and baby was discharged home. 


\section{Case 2}

A 2 month 23 day old male baby presented with history of fever for 1 day and painful swelling in the right groin. The baby was the first twin of twin siblings born at 34 weeks gestation by LSCS, was admitted in NICU for 48 hours for low birth weight and respiratory distress which settled with oxygen. Current weight was $4.43 \mathrm{~kg}$. Family history was insignificant. On examination there was a swelling in the right inguinal region which was tender, with overlying erythema. On ultrasound there was a hypoechoic lesion in the right inguinal region suggestive of nodal abscess. Blood investigations showed only mildly elevated CRP of 1.2. Incision and drainage were done and i.v. Meropenem and Teicoplanin was given with which he improved clinically. Blood culture was negative. Pus culture was also sterile. Baby was discharged after 7 days of antibiotic therapy.

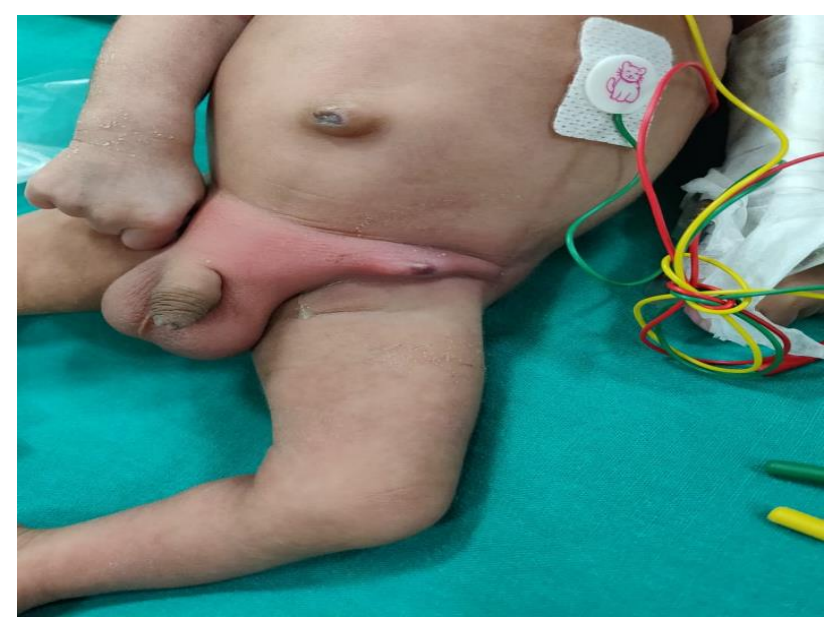

Figure 1: Left groin swelling with visible pus pointing.

\section{DISCUSSION}

Inguinal abscess is uncommon in infants and children. ${ }^{1}$ It can be primary which is idiopathic or has no detectable source and secondary in which the source of infection is direct spread from adjacent structures. ${ }^{1}$ Majority of cases in the infantile period is primary. The proposed hypothesis in case of primary abscess is that the normal flora of the skin spreads haematogenously due to immature immune system in infants. ${ }^{1}$ The most common organism seen in primary inguinal abscess is Staphylococcus aureus. In case of secondary inguinal abscess common organisms implicated are Staphylococcus aureus, Streptococcus pneumoniae and Escherichia coli. ${ }^{1}$ In present case however Staphylococcus hominis was isolated in one of the twins which has not been reported in any of the cases reported previously in literature. Staphylococcus hominis may be a normal flora of the human skin, but these bacteria can also be pathogenic in skin and soft tissue infections. ${ }^{2}$

The major presenting symptoms include leg or groin swelling, limitation of leg motion and pain. ${ }^{3,4}$ They may present as a tender mass palpable in the iliac fossa, lower abdomen, pelvis or the inguinal region., ${ }^{3,4}$ Peritoneal irritation is uncommon since the fascia covering the psoas muscle prevents spreading of the abscess to retroperitoneum or free peritoneal space., ${ }^{4,5}$ Differential diagnosis includes septic arthritis of hip, osteomyelitis of femur, traumatic injury to hip joint and surrounding structures, and abscess at vaccination site. ${ }^{4,5}$ The most important differential diagnosis is septic arthritis of hip and a poor response to antibiotics should prompt a suspicion of inguinal abscess. It is important to note that septic arthritis can often cause secondary inguinal abscess. ${ }^{5}$ Imaging with ultrasound, computed tomography (CT), or MRI helps in differentiating the above differentials. ${ }^{3-5}$

Routine investigations may show neutrophilic leukocytosis or raised CRP. ${ }^{3,4}$ Ultrasound is the investigation of choice which confirms the diagnosis and delineates the areas of collection. Antibiotic alone is insufficient in most cases and drainage is required. ${ }^{3-5}$ Ultrasound guided percutaneous drainage is feasible; however open surgical drainage gives a better exploration and complete drainage. Primary inguinal abscess can present as an emergency simulating septic arthritis with septicemia. Delayed treatment and inadequate drainage carry a high risk of mortality and sequelae due to damage to the joint. ${ }^{3-5}$ Both of our cases underwent incision and drainage following which they had quick recovery.

Whether every case of neonatal inguinal abscess need workup for immunodeficiency is unclear as most of the reported cases have not undertaken it. However, there are cases of inguinal abscess leading to diagnosis of immunodeficiency. ${ }^{6}$ As of now any infant with primary inguinal abscess should be assessed for features of immunodeficiency such as failure to thrive, recurrent infections, and should be regularly assessed. ${ }^{6}$

In case of any evidence appropriate work up should be done. Both the twins are under follow up and are thriving well with no evidence of any immunodeficiency.

\section{CONCLUSION}

Our case therefore emphasized the fact that early diagnosis and prompt treatment of inguinal abscess leads to quick and complete recovery. In case of any evidence appropriate work up should be done. In our case both the twins are under follow up and are thriving well with no evidence of any immunodeficiency.

Funding: No funding sources

Conflict of interest: None declared

Ethical approval: Not required

\section{REFERENCES}

1. Horiuchi A, Kameoka K, Kuwabara J, Watanabe Y, Kawakami S, Tauchi H, et al. Neonatal iliopsoas abscess. Pediatr Int. 2012;54:712-4. 
2. Natsis NE, Cohen PR. Coagulase-negative staphylococcus skin and soft tissue infections. Am J Clin Dermatol. 2018;19(5);671-7.

3. Patela R, Pimpalwara A, Huttona K. Primary neonatal iliopsoas abscess. J Pediatric Surg Case Rep. 2013;1:11-3.

4. Al-Zaiem MM, Bajuifer SJ, Fattani MO, Al-Zaiem FM. Bilateral iliopsoas abscess associated with right hip septic arthritis in a neonate. Saudi Med J. 2014;35:743-6.

5. Okada Y, Yamataka A, Ogasawara Y, Matsubara K, Watanabe T, Lane GJ, et al. Ilio-psoas abscess caused by methicillin-resistant Staphylococcus aureus (MRSA): A rare but potentially dangerous condition in neonates. Pediatr Surg Int. 2004;20:734.

6. Karabayir N, Turel O, Aydogmus C, Hatipoglu N, Hocaoglu A, Adal E, et al. Iliopsoas abscess in the neonate with immunodeficiency. Pediatr Int. 2012;54:439-40.

Cite this article as: Sadhukhan M, Mishra BN, Joshi RK, Pahi PP. Inguinal abscess in twin infants. Int Surg J 2020;7:1704-6. 